\title{
SELECTED QUOTATIONS
}

"I took this highly conservative view of the matter." (H. C. A R P, not in text)

"I don't know how to make galaxies - that's my problem." (G. R. BUR BIDGE, p. 107)

"Interacting or colliding systems form an interesting subject of study, but are not new types of galaxies. (After a collision a car is a wreck, not a new type of car!" (G. DE VAuCOULEURS, p. 6)

"History has shown that the Universe is complex." (w. C. SASLAW, p. 332)

"I can't possibly swear that some sort of fissioning of galaxies might not - even write ALAR TOOMRE in the sky." (ALAR TOOMRE, p. 363)

"I don't want to be considered lacking in photographic skill so I would point out that I too have published pictures where the connection (between NGC 4319 and Markarian 205) was not present." (H. C. AR P, not in text)

"What generates the enormous energies that pour from galactic nuclei? How do they evolve? And are new physical laws needed to understand them? Even though I've only asked three questions so far, already the ratio of questions to answers is infinite." (w. c. SASLAw, p. 305)

"The result you get is so improbable that I would try any way of escape first." (J. H. OORT, p. 241)

'Philosophical questions cannot be answered!' (A. TоOMRE, p. 242)

"It must be a general phenomenon, and theoreticians should explain it." (G. DE VAUCOULEURS, p. 340) 